\title{
XLVIII. Optical rotation, optical isomerism, and the ring-electron
}

\section{H. Stanley Allen M.A. D.Sc.}

To cite this article: H. Stanley Allen M.A. D.Sc. (1920) XLVIII. Optical rotation, optical isomerism, and the ring-electron, Philosophical Magazine Series 6, 40:238, 426-439, DOI: $10.1080 / 14786441008636143$

To link to this article: http://dx.doi.org/10.1080/14786441008636143

曲 Published online: 08 Apr 2009.

Submit your article to this journal $[\pi$

Џ Article views: 4

Q View related articles $\square$

Citing articles: 2 View citing articles 5 
XLVIII. Optical Rotation, Optical Isomerism, and the RingElectron. By H. S'Tanley Allen, M.A., D.Se., Lniversity of Edinlurgh *.

\section{Introduction.}

W HEN a beam of plane-polarized light passes through certain pure liquids and even certain vapours, there is produced a rotation of the plane of polarization, which implies that there must be some asymmetry in the molecule of the substance concerned. This asymmetry has been studied mainly from the chemical side, the difference between isomeric molecules of the same structure being explained by a different arrangement of atoms in space. On the physical side progress has been less satisfactory, and although a formal connexion between optical rotation and the electromagnetic theory of light was established by Drude $\dagger$, it is only recently that successful attempts have been made to explain why asymmetry in molecular structure should involve difference in the velocity of propagation of circularly polarized light. In this paper the subject is approached from a somewhat different standpoint from that usually adopted, for it is assumed that the electron, in addition to its electrostatic action, behaves like a current circulating in a closed ring, and consequently acts as a small magnet.' This conception involves a modification of prevailing views, both as regards the action of light on a system of electrons, and also as regards the constitution of the molecule. The paper therefore divides naturally into two parts. The first is concerned with the propagation of an electromagnetic wave through an assemblage of ring-electrons, whilst the second (which may prove of more interest to the chemist) deals with the question of the arrangement of the electrons in the molecule of an optically active substance, assuming the theory of the "cubical atom" developed by G. N. Lewis $\ddagger$ and Irving Langmuir $\$$.

* Communicated by the Author.

+ Drude, 'The Theory of Optics,' part ii. Chapters vi. \& viii.

† G. N. Lewis, Journ. Amer. Chem. Soc. vol. xxxviii. p. 762 (1916). 1919).

\$ I. Langmuir, Journ. Amer. Chem. Soc. vol. xli. pp. 868, 1543 


\section{PAR'T I.}

\section{Optical Rotation.}

Rotation of the plane of polarization of light by a pure liquid or a vapour has presented serious difficulties to the theoretical physicist. Drude and Voigt have shown what type of electromagnetic equations are required to account for the rotation, but "there is no satisfactory representation of the mechanism by means of which an asymmetrical molecular structure turns the plane of polarization "*. In the ordinary theory of dispersion the equation of motion of an electron (mass $m$, charge $e$ ) is of the form

$$
{ }_{m} \frac{\partial^{2} \xi}{\partial t^{2}}=e \mathrm{X}-\frac{4 \pi \epsilon^{2}}{\theta} \xi,
$$

where $\xi$ is the $x$-component of the displacement from the equilibrium position, $\mathrm{X}$ is the $x$-component of the exterior electric force, and the last term represents the restoring force called into play by the displacement of the electron. Drude includes also a frictional term which represents a force retarding the vibrations. In an isotropic medium the only possible extension of the equation is by the introduction of a term of the form ef'( $\left.\frac{\partial \mathrm{Y}}{\partial z}-\frac{\partial Z}{\partial y}\right)$. By combining the equations thus modified with Maxwell's equations for the electromagnetic field, it can be shown that when planepolarized light falls on the medinm, two waves are propagated through it with different velocities, the first representing right-handed cireular polarization, the second left-handed circular polarization. The superposition of the two waves yields a plane-polarized wave in which the plane of polarization rotates through a definite angle for each unit length of optical path. The terms which have been added to the equations may be taken to represent a torsional electric force. Drude gives a graphical representation by conceiving that because of the molecular structure the paths of the electrons are not short straight lines, but short belices twisted in the same direction, with their axes directed at random in space. "A riffe bullet lying in its rifle barrel would be displaced in a similar manner along the barrel both by a pulling and twisting force. But if we take the dimensions of a single electron to be very small, we exclude the possibility of a constraint which would enable a couple to cause a motion in one direction. We must in that case draw the conclusion

* Schuster, 'The Theory of Optics,' § 162, 1904 edition.

$2 \mathrm{~F} 2$ 
that the vibrations of the electron which give rise to the rotatory effect are motions of systems of electrons united together by certain forces which are such that a couple of electric forces produces a displacement of the positive electrons in one direction or of the negative electrons in the opposite direction along the axis of the couple" (Schuster).

In the last few years several mathematical physicists, notably Born * and Gray $\dagger$, have developed the latter hypothesis, and have shown that the optical activity of liquids and gases can le explained by regarding the molecules as coupled systems. Born views a molecule as a system of coupled electrons, the coupling and the restoring forces being identical. In the paper by Gray the atom is looked upon as a particle of dielectric. The atoms are coupled according to the ordinary laws for a doublet, and the restoring force on an electron is not identical with the coupling, but may be influenced by it.

To the present writer it appears that a simpler and more realistic mental picture of optical activity may be obtained by abandoning the limitation that the dimensions of the electron must be very small, and employing the ring-electron or "magneton" of A. L. Parson $\neq$. Such an electron vibrating backwards and forwards along a straight line seems admirably adapted to replace the electron moving in a spiral path as imagined by Drude.

\section{The Ring-Electron.}

The ring-electron may be looked upon as a charge of negative electricity distributed continuously around a ring: which rotates on its axis with high speed, and therefore lebaves like a small magnet. In an important paper on the electromagnetic mass of the Paison magneton, Webster \&. has shown that the ratio of the radius of the cross-section of the ring to that of the ring itself is extremely minute, and that most of the energy and momentum of the field are concentrated very closely around the ring. Thus, to a first approximation, the ring-electron may be segarded as a current in a circular wire of negligible thickness. In a later paper $\|$ it is shown that the gyroscopic effect of the magneton

* Born, Phys. Zeitschr. vol. xri. p. 251 (1915); Ann. der Physit, rol. lv. p. $17 \%$ (1918).

+ Gray, Phys. Rev. rol vii. p. 472 (1916).

†. A. L. Parson, "A Magneton Theory of the Structure of the Atom," Smithsunian Misc. Coll. No. 2371, Nor:" 1915.

§ Webster, Pbys. Rev. vol. ix. p. 484 (1917).

II Webster, Phỵs. Rev. vol. ix. p. 561 (1917). 
is exactly the same as for an electron of the classical type moving in an orbit equal in size to the ring with a speed equal to that of the electricity of the ring. These results simplify very greatly the consideration of problems connected with the ring-electron.

In the case of an ordinary electron exposed to light, the incident vibrations of the light bring about forced ribrations through the action of the electric vector in the wave-front. With the modification here proposed, the effects are more complicated, for the ring-electron will be acted upon both by the electric and magnetic vectors. Any rotation of the plane of the ring, which may be produced by the magnetic force of the light-wave, will be neglected. There is a more important effect due to the alternating electromotive force acting round the ring and producing changes in the magnetic moment of the equivalent magnet. Consider a fixed ringelectron, the axis of the ring being parallel to the axis of $x$. When this is exposed to a light-wave, there will be an electromotive force * in the ring given by

$$
\begin{aligned}
\mathrm{E} & =-\frac{d}{d t} \iint_{\int} \alpha d \mathrm{~S}=-\iint c\left(\frac{\partial \mathrm{Y}}{\partial z}-\frac{\partial \mathrm{Z}}{\partial y}\right) d \mathrm{~S} \\
& =-\mathrm{A} c\left(\frac{\partial \mathrm{Y}}{\partial z}-\frac{\partial \mathrm{Z}}{\partial y}\right), \text { approximately, }
\end{aligned}
$$

where $A$ is the area of the ring. If this alternating E.M.F. be represented by $\mathrm{E}_{0} \cos p t$, there will be round the ring an induced current differing in phase by $\frac{\pi}{2}$ from the electromotive force, and represented by $\frac{E_{0} \sin p t}{\mathrm{~L}_{p}}$. The mannetic moment of the electron will be increased by an amount $\frac{\mathrm{AE}_{0} \sin p t}{\mathrm{~L} p}$, and in consequence there will be a mechanical force acting upon the electron proportional to this increase and to the space variation of the controlling magnetic field. Thus the equation of motion of the electron will contain a term of the form $e f^{\prime \prime}\left(\frac{\partial Y}{\partial z}-\frac{\partial Z}{\partial y}\right)$ required by Drude's theory, the coefficient $e f^{\prime}$ being proportional to $A^{2} / \mathrm{L}$, and depending also on the character of the magnetic field due to the remainder of the molecule or to any external magnetic system. It then follows, as shown by Drude, that there will be two

* Following Drude, E, $a, \beta, \gamma$ are in electromagnetic units, whilst $e, X, Y, Z$ are in electrostatic units. 
circularly polarized waves travelling through the mediunf with different velocities, according as the rotation is righthanded or left-handed, and in consequence the plane of polarization will rotate uniformly about the direction of propagation of the light, the amount of the rotation per unit length being

$$
\delta=2 \pi^{2} f / \lambda^{2},
$$

where $\lambda$ is the wave-length of the light (in vacuum),

and

$$
f=\Sigma \frac{\theta f^{\prime} \mathrm{N}}{1-\left(\begin{array}{c}
\tau_{h} \\
\tau
\end{array}\right)^{2}} .
$$

Here $\mathrm{N}$ denotes the number of electrons of the type considered, in unit volume, $\tau$ is $1 / 2 \pi$ times the period of vibration of the light, and $\tau_{h}$ is $1 / 2 \pi$ times the natural free period of the electron.

It is not elaimed that the foregoing discussion gives a complete account of the behaviour of ring-electrons under the influence of light-waves. There are other actions which may be briefly referred to. In connexion with the magnetic rotation of the plane of polarization, Drude has given a theory based on the hypothesis of molecular currents, as conceived by Ampere and Weber. It is assumed that these molecular currents are made parallel to one another by the action of the external field. Drude points out that the displacement of the molecular current when a light-wave falls upon it, produces a displacement of the magnetic lines of force which arise from it, so that a peculiar induction effect takes place. It is to be observed that this theory calls for rotations of opposite sign on opposite sides of an absorption band. It would seem that a complete theory of the action of lightwaves on an assemblage of ring-electrons would have to take into consideration also the "Hall effect," which has been found to yield a satisfactory explanation of rotatory dispersion in a magnetic field. Thus the hypothesis of the Hall effect explains the result that the rotation is in the same direction on opposite sides of the absorption band in the case of sodium vapour, and also predicts an effect when the rays of light are perpendicular to the lines of magnetic force. I have discussed elsewhere * the possibility of accounting for the Zeeman effect by means of the ring electron.

* H. S. Allon, Proc. Phys. Soc. Lond, vol. xxxi. p. 49 (1919). 


\section{PART II. \\ Optical Isomerism.}

In the theory of atomic structure first put forward by G. N. Lewis and afterwards developed by Langmuir, the electrons, instead of rotating in rings as in Bohr's theory, are supposed to occupy, or to oseillate about, positions which are fixed in space with reference to the atomic nucleus. This fixity of the electrons is a characteristic feature of the magneton theory of the atom advanced by Parson-a theory which has not met with the recognition it merited, partly because it is based on the notion of a positive sphere, partly because Parson did not accept the atomic numbers of Moseley, which are now regarded as being determined not merely in a relative but also in an absolute sense. The magneton, or ring-electron, makes it possible to have stationary electrons. The most stable groupings of electrons, according to Lewis and Langmuir, are (1) the pair, as illustrated in the helium atom, (2) the octet, or group of eight electrons arranged approximately at the corners of a cube. Parson showed that such a group of eight magnetons formed a system possessing very low magnetic energy and producing a very weak external field. This assumption is in agreement with the "rule of eight," to which I have drawn attention in connexion with atomic and molecular numbers*. The number of unit electric charges in the atomic nuclei of related atoms or molecules frequently differs by 8 or a multiple of 8 .

In the formation of chemical compounds only an even number of electrons can be held in common. "Two octets may hold 1,2 or sometimes even 3 pairs of electrons in common. A stable pair and an octet may hold a pair of electrons in common. An octet may share an even number of its electrons with $1,2,3$ or 4 other octets. No electrons

Fig. 1.

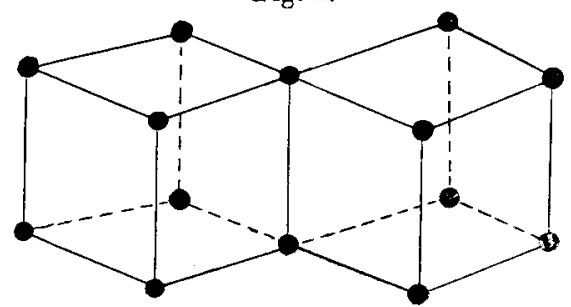

can form parts of more than two octets" (Langmuir, Postulate 11). Thus the single bond commonly used in graphical formulæ involves two electrons held in common by two atoms (fig. 1); the double bond implies that four electrons

* H. S. Allen, Trans. Chem. Soc. vol. cxiii. p. 389 (1918). 
are held conjointly by two atoms (fig. 2). According to a suggestion made by Lewis and adopted by Langmuir, the electrons, which are held in common between two octets or an octet and a stable pair, are drawn together to form pairs,

Fig. 2 .

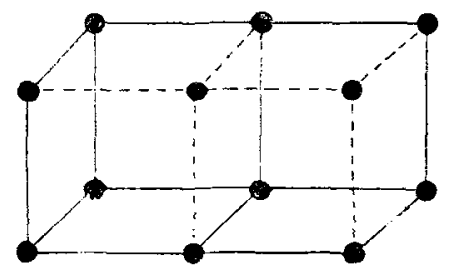

perhaps by the action of magnetic forces. Thus in methane $\left(\mathrm{CH}_{4}\right)$ the 8 electrons are located in pairs at the 4 corners of a tetrahedron, each hydrogen nucleus being held by one pair.

On this view, dextro- and lævo-rotatory forms of a compound may be represented, as I have pointed out previously*, by mirror images as in fig. 3. In this diagram the letters $\hat{N}$ and $S$ may be supposed to indicate the polarity of the exposed face of the ring-electron.

Fig. 3.
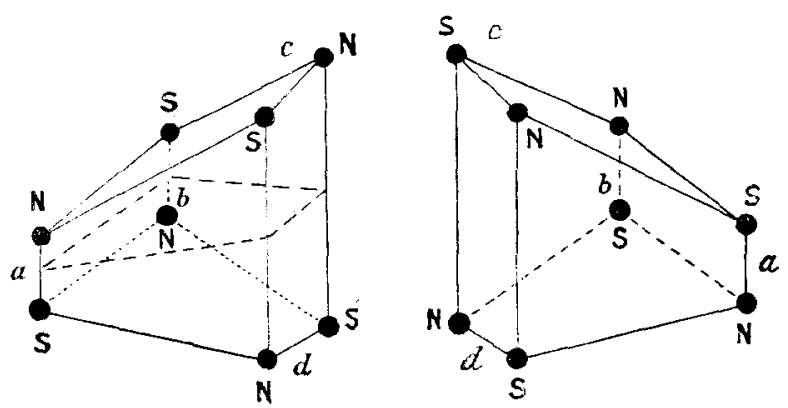

A suggestion has been made by W. E. Garner that a large number of optical isomerides may possibly exist amongst organic compounds in consequence of the right-handed or

* 'Nature,' vol. cv. p. 71 (1920).

+ W. E. Garner, 'Nature,' vol. civ. p. 661 (1920); vol. cv. p. 171 (1920). See also letters from A. E. Oxley, id. vol. cv. pp. 105, 231 (1920). 
left-handed rotation of a valency electron around the direction of a chemical bond. This suggestion does not receive support from the arrangement shown in the figure which seems to yield exactly the same number of isomerides as the ordinary structural formula. It is true thint it is possible to reverse in the diagram the magnetic polarity of one or more pairs of electrons, but even if the arrangements so obtained were stable, it is doubtful whether they would represent different isomerides. If such a reversal of the magnetic polarity were accompanied by a change in the nature of the compound, it does not seem possible to explain the phenomenon of free mobility about a single bond which is assumed in stereochemistry. It should, however, be mentioned that although the prevailing view is that the single bond between carbon atoms does not fix the positions of the atoms comnected by it as regards rotation about the common axis, the contrary opinion has been supported by Aberson (Cohen, 'Organic Chemistry for Advanced Students,' pp. 116, 133).

This raises a question in the theory of the cubical atom which requires further elucidation. It is clear from fig. 3 that the electrons associated with group $a$ are related to the electrons of group $b$ in a manner different from that in which they are related to the electrons of $c$ or $d$. Lewis assumes that each pair of electrons is drawn together so as to represent a single corner of "the model of the tetrahedral carbon atom which has been of such signal utility throughout the whole of organic chemistry." But even if the electrons in a pair are drawn closer together than in the diagram, the lines joining the centres of the pairs are oriented differently when $a$ and $b$ are compared with $c$ and $d$. The difficulty might be got over by supposing the electrons in a pair to rotate about a point midway between them, or in the case of the ring-electron one electron might be supposed to move over the other, giving the arrangement

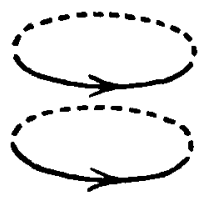

instead of

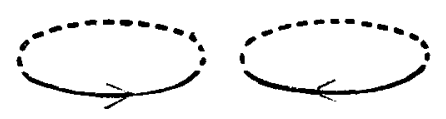

1)r. Langmuir has been kind enough to express his views on this question in a letter to the author. "When a pair of electrons acts as a bond between two adjacent atoms, the relationship between the two electrons has certainly becom. changed. The very fact that we never have one or three 
electrons held in common between two atoms is proof that the electrons in the pair are bound into a kind of unit, in which the relitionship between the two electrons is very different from that between two adjacent electrons in the neon atom .... In practically all the compounds of carbon, there are eight electrons arranged around the kernel of the carbon atom, but these electrons are gathered into four pairs, each pair constituting a $u_{i i i t}$ or bond between the carbon atom and atoms surrounding it. The nature of this unit is evidently closely related to that of the pair of electrons in the helium atom and hydrogen molecule, and my guess is that such a pair consists of two electrons revolving about a line comnecting the kernels of the adjacent atoms, whereas the electrons in the neon atom are revolving about eight positions located at tle corners of a cube. We are thus led to a conception of the carbon atom which is practically identical with that of the organic chemist, namely: that the carbon atom exhibits four valence bonds arranged in space in a symmetrical way, corresponding to the corners of a tetrahedron."

According to the present theorr, optical activity arises from a difference effect, and can be manifested only when there is lack of compensation amongst the electrons associated with the various parts of the molecule. If the chemical bond is to he attributed to a pair of electrons arranged side by side, it is easy to understand how such compensation can he brought about in the great majority of chemical compounds. In the case of a single asymmetric carbon atom. the symmetrical arrangement of each of the four electron pairs is disturbed hy the presence of the adjacent groups, resulting in only partial compensation. Thus, in the compound C a b c d, the pair of electrons associated with group $a$ is under the influence of the unlike groups $c$ and $d$, and the condition of symmetry is absent. But if $c$ and $d$ are made alike, the whole molecule will have a plane of symmetry indicated by the broken line in the left hall of fig. 3. Thus the molecule will be inactive through " internal compensation" with respect to the electrons which form the outer shell of the carbon atom.

This arrangement of the electron pair side by side gives readily an explanation of the fact that most chemical compounds show diamagnetic properties, for such a pair would produce a very weak external magnetic field, and this configuration has in fact been utilized by Oxley * to account for the diamagnetism of the hydrogen molecule.

* 'Oxley, 'Nature,' vol. cv. p. 327 (1920). 
The evidence at present availabie does not justify a decisive verdict as between the two possible arrangements
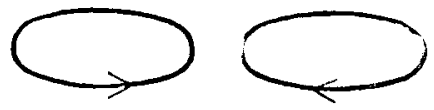

and

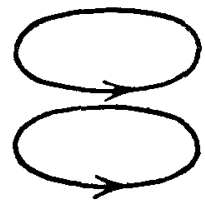

for the electron pair. It may be that both are possible, with corresponding differences in the optical and magnetic properties of the compound. On the basis of the latter configuration, a large number of isomerides may exist in accordance with the suggestion of Garner, referred to above. "The electrons rotating in pairs around the four carbon valencies may possess either clockwise or anti-clockwise rotation with respect to the central carbon atom. On the assumption that two of these pairs of electrons rotate in a clockwise and two in an anti-clockwise direction, it is possible to deduce that eight isomerides of cinnamic acid may exist." If the clockwise rotation of the electron gives a north-seeking character to the valency and the anti-clockwise rotation a southseeking character, isomerides may exist which may be represented graphically as below
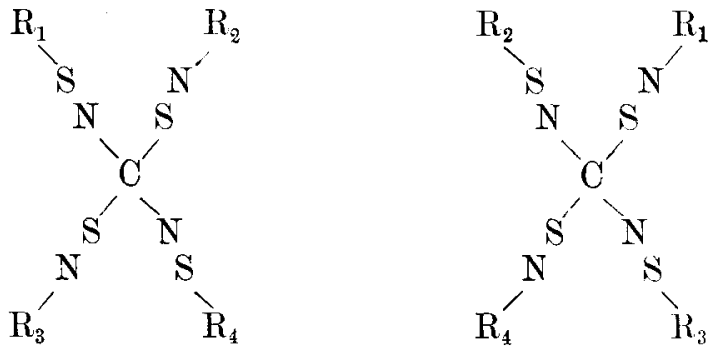

The existence of the isomeric compounds suggested by Garner depends upon the somewhat arbitrary assumption that two of the pairs of electrons are rotating in a clockwise sense, and the other two in an anti-clockwise sense as viewed from the carbon atom. In a three-dimensional model the four valencies may be supposed directed towards the corners of a regular tetrahedron. It will then be observed that Garner's assumption implies that the valency electrons in such a compound confer upon it paramagnetic properties, since the suggested arrangement of electrons would have 
a resultant magnetic moment. It would, of course, be possible to overcome this difficulty by a further assumption that magnetic compensation arises from other parts of the molecule.

\section{Optical Rotatory Power.}

The theory of optical rotation for liquids and gases here proposed appears to be in good agreement with the experimental facts as summarized by Gray.

1. Any substance of which the molecules are truly asymmetrical causes rotation.

2. Antipodes, a molecule and its mirror image, rotate in opposite directions.

3. Symmetrical molecules are not active.

t. Absorption systems in an active substance cause anomalies in the rotary dispersion, although the origin of the band may lie in a part of the molecule distant from the central carbon atom.

5 . The magnitude of the activity is profoundly influenced by the molecular structure, and is roughly dependent on the magnitude of the asymmetry of the molecule.

6. Activity varies with temperature and pressure, and with the concentration of active molecules.

From what has already been said it is obvious that the first three results are in accord with the theory. The fourth result follows alike from the electrostatic theories of Born and of Gray and from the electromagnetic theory here given. In the first case the rotation depends on a space derivative of the electric force, in the second on a space derivative of the magnetic force. The fifth result requires somewbat closer examination.

Guye * and Crum Brown $\dagger$ have discussed independently the relation of optical activity to the character of the radicles united to the asymmetric carbon atom. "It is obvious that the amount of optical activity of a given compound containing an asymmetric atom of carbon depends upon the amount of difference in character among the four radicles united to the asymmetric carbon atom, so that if two of them are very nearly equal we come very near to a compound of a symmetric carbon atom, in which the optical activity is zero. The question suggests itself, How are we to measure this difference of character?" Guye regarded

* Guye, Compt. Rend. vol. cx. p. 714 (1890) ; vol. cxvi. pp. 1378, $1451(1893)$.

† Crum Brown, Proc. Roy. Soc. Edin. vol. xvii. p. 181 (1890). 
the mass of each radicle and the distance of its centre of gravity from the centre of figure of the tetrahedron as all that need be considered-a view which conld not be held at the present time; but Crum Brown assumed merely "that there is a function, capable of numerical representation, derivable from the composition and constitution of the radicle and the temperature of the substance, and that it is the difference between the values of this function in the case of two radicles which gives us the difference of character roferred to." According to the present theory, it would he the values of the magnetic and electric fields produced by the radicles which would determine the degree of asymmetry.

Amongst the papers read at the discussion on "Optionl Rotatory Power;" held before the Fariday Society in 1914, one of the most interesting from a theoretical standpoint was that by Len Tschugaeft. He came to the conclusion that the electrons which are most active in producing rotation are attached to the asymmetric carbon atom itself, or are situated in the immediate neighbourhood of the centre of activity. As regards the other electrons which come into play in rotatory dispersion, we can assume that their activity is liminished with iucreasing distance from the asymmetric complex. Again, if it be admitted that the degree of asymmetry of the molecule depends upon the differences of four constants $K_{1}, K_{2}, K_{s}$, and $K_{4}$, corresponding with the four groups attached to the carbon atom, the value of the unknown function $\mathrm{K}$ must depend on the degree of saturation. "Thus, it would be expected that strongly unsaturated radicles containing free-movable electrons would exert a considerable influence on the electric field produced br the molecule, the differences $\mathrm{K}_{1}-\mathrm{K}_{2} \ldots$ being much larger if $\mathrm{K}_{2}$ corresponds with a saturated group and $\mathrm{K}_{1}$ to an unsaturated one than if both the groups are nearly equally siturated."

The present theory is in general agreement with the views just described, but the equation representing the views of Guye and Crum Brown would require some modification. Since the rotatory power as well as the dispersion is assumed to be equal to the sum of the effects produced by the several active electrons, as is expressed by the formula

$$
\delta=\frac{l}{\lambda^{2}} \Sigma \frac{\theta f^{\prime} \mathrm{N}}{1-\left(\begin{array}{c}
\tau_{h} \\
\tau
\end{array}\right)^{2}},
$$

it is necessary to consider the contributions from the four pairs of electrons associated with the four attached groups. 
If the arrangement represented in fig. 3 be adopted, the asymmetry of pair $a$ is due to groups $c$ and $d$, and the resulting contribution to the rotation will he equal to the algebraic sum of two terms of the type given above. In order to explain the absence of rotation in a symmetrical molecule, it is necessary to assume that the sign is positive for one electron and negative for the second electron of the pair. Let us further assume, at least as a first approximation, that the natural tree period is the same for each electron, so that the denominator $1-\left(\frac{\tau_{h}}{\tau}\right)^{2}$ is the same. The contribution to the rotation made by the pair of electrons may then be written

$$
\begin{aligned}
\delta_{a} & =\frac{k}{\lambda^{2}} \frac{\theta \mathrm{N}}{1-\left(\frac{\tau_{h}}{\tau}\right)^{2}}\left(f^{\prime}{ }_{a_{1}}-f^{\prime \prime}{ }_{a_{2}}\right) \\
& =\mathrm{A}\left(f^{\prime}{ }_{a_{1}}-f^{\prime}{ }_{a_{2}}\right) .
\end{aligned}
$$

Hence the total result may be written

$$
\mathrm{P}=\mathrm{A}\left(\mathrm{K}_{\mathrm{c}}-\mathrm{K}_{d}\right)+\mathrm{B}\left(\mathrm{K}_{d}-\mathrm{K}_{c}\right)+\mathrm{C}\left(\mathrm{K}_{a}-\mathrm{K}_{b}\right)+\mathrm{D}\left(\mathrm{K}_{b}-\mathrm{K}_{a}\right),
$$

since the difference between $f^{\prime \prime} a_{1}$ and $f^{\prime \prime} a_{2}$ is due to the presence of the unlike groups $c$ and $d$. It follows that

$$
\mathrm{P}=(\mathrm{A}-\mathrm{B})\left(\mathrm{K}_{c}-\mathrm{K}_{d}\right)+(\mathrm{C}-\mathrm{D})\left(\mathrm{K}_{a}-\mathrm{K}_{b}\right) \text {. }
$$

In the case in which the groups $c$ and $d$ become identical, $\mathrm{K}_{c}=\mathrm{K}_{d}$ and $\mathrm{C}=\mathrm{D}$, so that there is no resultant activity.

If, on the other hand, the alternative arrangement of coaxial rings be adopted, the resultant effect would have to be calculated by methods sinilar to those employed by Gray* in the case of purely electrostatic forces.

According to the principle of optical superposition formulated by van't Hoff, the total rotatory power in a compound containing several asymmetric carbon atoms is the algebraic sum of the various radicles taken separately. This rule seems to be valid at least to a first approximation $\dagger$. The work of C. S. Hudson $\ddagger$ and his collaborators shows that the principle holds fairly closely in the case of certain amides. The approximate validity of the rule is to be expected on such a theory of optical rotation as that here put forward.

The variation of optical activity with temperature,

* Luc, cit.

+ Tschngaeff, loc. cit.

f C. S. IIudson, Journ. Amer. Chem. Soc. vol. xli. pp. 1140, 1141 (1919). 
pressure, and concentration presents difficulties whatever view may be adopted as to the origin of the rotation. The subject has been discussed by Livens *, who has examined the effect of the presence of inactive substances, as in the case where a simple active substance is dissolved in an inactive liquid, and also by Gray $\dagger$. Assuming that the atoms are vibrating about points of equilibrium inside the molecule, the average value of both the electrostatic and the magnetic field produced will change with the amplitude of the oscillations, and consequently the degree of asymmetry will be a function of the temperature.

Finally, it may be noted that the optical activity of compounds containing asymmetric atoms other than carbon atoms follows at once from the theory. Such active compounds are known in the case of nitrogen, phosphorus, sulphur, selenium, tin, silicon, cobalt, chromium, rhodium, and iron $\ddagger$. It is perhaps not without significance that the three elements cobalt, rbodium, and iridium occupy a peculiar position in Langmuir's theory, as in each case there is an odd clectron in the outer shell which is assumed to occupy a position at one end of the polar axis.

\section{Summary and Conclusion.}

In the present paper a theory of optical rotation has been advanced in which the electron, instead of being regarded as a point charge, is looked upon (as suggested by A. L. Parson) as an anchor ring of negative electricity rotating rapidly about its axis. Such a ring-electron vibrating in a linear path takes the place of an ordinary electron moving in a spiral path as postulated by Drude. It is shown that rotation of the plane of polarization of liglst will result, and an expression is found for the amount of rotation per unit length. Employing the theory of atomic structure due to Lewis and Lingmuir, a graphieal representation may be obtained for dextro- and lævo-rotatory forms of a compound. The experimental facts with regard to optical activity are in good agreement with the theory put forward, which may be applied not merely to carbon compounds, but to any compound containing an asymmetric atom.

* Livens, Phil. Mag. vol. xxv. p. 817 (1913).

+ Loc. cit.

† A. W. Stewart, 'Stereochemistry', chapter x. (1919). 